\title{
The Housing Problem and Revealed Preference Theory: Duality and an Application
}

\author{
Ivar Ekeland \\ (Université Paris-Dauphine)
}

\author{
Alfred Galichon \\ (Ecole polytechnique)
}

August 28, 2012*

\begin{abstract}
This paper exhibits a duality between the theory of Revealed Preference of Afriat and the housing allocation problem of Shapley and Scarf. In particular, it is shown that Afriat's theorem can be interpreted as a second welfare theorem in the housing problem. Using this duality, the revealed preference problem is connected to an optimal assignment problem, and a geometrical characterization of the rationalizability of experiment data is given. This allows in turn to give new indices of rationalizability of the data, and to define weaker notions of rationalizability, in the spirit of Afriat's efficiency index.
\end{abstract}

JEL codes: primary D11, secondary C60, C78.

Keywords: revealed preferences, Afriat's theorem, optimal assignments, indivisible allocations.

\footnotetext{
${ }^{*}$ Correspondence: Economics Department, Ecole polytechnique, 91128 Palaiseau, France. E-mail: alfred.galichon@polytechnique.edu. Support from Chaire EDF-Calyon "Finance and Développement Durable" and FiME, Laboratoire de Finance des Marchés de l'Energie (www.fime-lab.org) is gratefully acknowledged by both authors, and from Chaire Axa "Assurance et Risques Majeurs," Chaire FDIR "Socially Responsible Investments and Value Creation" by the second author. The authors thank John Geanakoplos for communicating them his paper "Afriat from MaxMin". This paper has benefited from insightful comments by Don Brown, Federico Echenique, Françoise Forges, Peter Hammond, Vincent Iehlé and Enrico Minelli.

This is a pre-print of an article published in Economic Theory. The final authenticated version is available online at: https://doi.org/10.1007/s00199-012-0719-x
} 


\section{Introduction}

The "Revealed Preference" problem and the "Housing problem" are two classical problems in Economic Theory with for both a distinguished, but separate tradition. This paper is about connecting them, and exploit the connection to obtain new results on Revealed Preference.

- The Revealed Preference (RP) problem, posed by Samuelson at the end of the 1930's, was solved by Houthakker in 1950, and was given an operational solution by Sidney Afriat in 1967. This classical problem asks whether, given the observation of $n$ consumptions baskets and corresponding prices, one rationalize these consumptions as the consumption of a single consumer facing different prices.

- The "housing problem" was investigated in 1974 by Shapley and Scarf. Given an initial allocation of $n$ houses to $n$ individuals, and assuming individuals form preferences over houses and can trade houses, what is the core of the corresponding game? It is assumed that houses form no preferences over owners (in sharp contrast to the "Stable matching" problem of Gale and Shapley). In this setting, they showed non-emptiness of the core, as well as an algorithm to arrive to a core allocation: the method of "top-trading cycles", attributed to David Gale.

Although both problems have generated two well established and distinct literatures, it will turn out that these problems are in fact dual in a precise sense. As we shall argue, the traditional expenditure/utility duality from consumer theory extends to the setting of revealed preference, and it is possible to show that the problem of Pareto efficiency in the housing problem and the issue of rationalizability of consumer data are both applications of a basic mathematical result characterizing cyclically consistent matrices proven by Fostel, Scarf and Todd (2004), generalizing Afriat's theorem. In particular, we show that Afriat's theorem can be interpreted as a second welfare theorem in the housing problem. Once we have established equivalence of both problems, we shall use the aforementioned results to give new characterization of both problems in terms of an optimal allocation problem. This will give us a very simple and intuitive characterization of the efficient outcomes in the indivisible allocation problem.

According to standard revealed preference theory, the data are not rationalizable when one can exhibit a preference cycle based on the direct revealed preference relation. A stronger violation of rationalizability occurs when there are subsets of the data where cycles exist. An even stronger departure from rationalizability occurs when all observations are part of such a cycle. We shall characterize these three situations in terms of indexes which will measure how strong the violations of rationalizability are. In the last part, we shall last use these results to provide a geometric interpretation of revealed preference theory, by showing that the problem of determining whether data can be rationalized or not can be recast as the problem of determining whether a particular point is on some part of the boundary of some convex body. 
The connection between the housing problem and the revealed preference problem yields some insights for both theories. The important econometrics literature on revealed preference contains a wealth of tools to measure the intensity of departure from rationalizability. These tools may be successfully applied to the "housing problem" and its more applied versions such as the "kidney problem", which deals with assigning organ transfers to patients. These tools may design indices to measure how far from Pareto optimality an actual assignment is, or what are the costs of a given constraint in terms of welfare. Conversely, the connection between the two problems sheds light on the role of matching theory into revealed preference theory. This leads to interesting research avenues, as matching theory has recently received a lot of attention, both from the empirical and computational point of views.

The literature on revealed preference in consumer demand traces back to Samuelson (1938), who formulated the problem and left it open. It was solved by Afriat (1967) using nontrivial combinatorial techniques. Diewert (1973) provided a Linear Programming proof and Varian (1982) an algorithmic solution. Fostel, Scarf and Todd (2004) and Chung-Piaw and Vohra (2003) provided alternative proofs. Matzkin (1991) and Forges and Minelli (2009) extended the theory to nonlinear budget constraints. Geanakoplos (2006) gives a proof of Afriat's theorem using a minmax theorem. Although min-max formulations will appear in our paper, these are distinct from Geanakoplos', as it will be explained below. The setting was recently extended to collective models of household consumptions by Cherchye, De Rock and Vermeulen in a series of paper (see e.g. 2007, 2010). A very recent paper by Quah (2012) extends Afriat's Theorem in another direction $\mathrm{n}$ order to characterize data sets compatible with weak separability without concavity assumptions. The literature on the indivisible allocation problem was initiated by Shapley and Scarf (1974), who formulated as the "housing problem" and gave an abstract characterization of the core. Roth et al (2004) study a related "kidney problem" and investigate mechanism design aspect. Finally, recent literature has extended revealed preference theory to classes of matching problems. Galichon and Salanié (2010) investigate the problem of revealed preferences in a matching game with transferable utility, with and without unobserved heterogeneity. Echenique et al. (2011) investigate the problem of revealed preferences in games with and without transferable utility, and without unobserved heterogeneity.

This paper will be organized as follows. We first study Pareto efficient allocations, and show the connection with the generalized theory of revealed preferences. We deduce a novel characterization of data rationalizability in terms of an optimal matching problem, and a geometric interpretation of revealed preference in terms of convex geometry. We then move on to providing indexes of increasingly weaker forms of rationalizability. 


\section{Revealed preference and the housing problem}

\subsection{The Generalized Afriat's theorem}

Assume as in Forges and Minelli (2009) that consumer has budget constraint $g_{i}(x) \leq 0$ in experiment $i$ where $x$ is an $L$-dimensional bundle of goods, and that bundle $x_{i}$ is chosen where $g_{i}\left(x_{i}\right)=0$. This is a nonlinear generalization of Afriat (1969), in which $g_{i}\left(x_{j}\right)=x_{j} \cdot p_{i}-x_{i} \cdot p_{i}$, where $p_{i}$ is the price vector of the bundle of goods in experiment $i$. Forges and Minelli ask whether there exists a utility function $v(x)$ with appropriate properties such that

$$
x_{i} \in \operatorname{argmax}_{x}\left\{v(x): g_{i}(x) \leq 0\right\}
$$

in which case $v$ is said to rationalize the data.

As in the original Afriat's paper, the first part of their proof necessitates the existence of a utility level $v_{j}$ associated to bundle $j$ such that

$$
g_{i}\left(x_{j}\right)<0 \text { implies } v_{j}-v_{i}<0 .
$$

Once the number $v_{i}$ 's have been determined, the second part of their proof consists in constructing a locally nonsatiated and continuous utility function $v(x)$ such that $v\left(x_{j}\right)=v_{j}$. The preference induced by $v$ are said to rationalize the data in the sense that

$$
i \in \operatorname{argmax}_{j}\left\{v_{j}: g_{i}\left(x_{j}\right) \leq 0\right\} .
$$

The existence of real numbers $v_{i}$ such that (1) holds is non-trivial and it turns out to be equivalent to a property of matrix $R_{i j}=g_{i}\left(x_{j}\right)$ called "cyclical consistency". This is done by appealing to an extension of Afriat's original theorem beyond linear budget sets, proved by Fostel, Scarf and Todd (2004). This result can be slightly reformulated as follows:

Theorem 0 (Afriat's theorem). The following conditions are equivalent:

(i) The matrix $R_{i j}$ satisfies "cyclical consistency": for any cycle $i_{1}, \ldots, i_{p+1}=i_{1}$,

$$
\forall k, R_{i_{k} i_{k+1}} \leq 0 \text { implies } \forall k, R_{i_{k} i_{k+1}}=0,
$$

(ii) There exist numbers $\left(v_{i}, \lambda_{i}\right), \lambda_{i}>0$, such that

$$
v_{j}-v_{i} \leq \lambda_{i} R_{i j},
$$

(iii) There exist numbers $v_{i}$ such that

$$
\begin{aligned}
& R_{i j} \leq 0 \text { implies } v_{j}-v_{i} \leq 0, \text { and } \\
& R_{i j}<0 \text { implies } v_{j}-v_{i}<0 .
\end{aligned}
$$


Before we give the proof of the result, let us briefly comment on it. Although we call it "Afriat's theorem", this result is in some sense a fundamental lemma upon which Afriat's analysis rests. In the original Afriat's work and in the subsequent literature, notably Forges and Minelli (2009), a full description of the budget sets is provided, that is the functions $g_{i}(x)$ defined on a larger domain than the set of observed consumption bundles $\left\{x_{1}, \ldots, x_{n}\right\}$. Here, the result that we give only depends on the value $g_{i}\left(x_{j}\right)$ of the functions $g_{i}$ over the set of the observed consumption bundles. Actually, even only the signs of these values matter. Here the analysis is intrinsiquely discrete and the approach relies neither on a particular consumption space, nor on explicit consumption bundles $x_{j}$.

We now turn to the proof of Theorem 0 .

Proof of Theorem 0. (i) implies (ii) is proven in Fostel, Scarf and Todd (2004). (ii) immediately implies (iii). We now show that (iii) implies (i). Consider a cycle $i_{1}, \ldots, i_{p+1}=i_{1}$, such that

$$
\forall k, R_{i_{k} i_{k+1}} \leq 0 \text {. }
$$

Then by (iii) there exist numbers $v_{i}$ such that

$$
\begin{aligned}
& R_{i j} \leq 0 \text { implies } v_{j}-v_{i} \leq 0, \text { and } \\
& R_{i j}<0 \text { implies } v_{j}-v_{i}<0 .
\end{aligned}
$$

thus one has $v_{i_{k+1}}-v_{i_{k}} \leq 0$ for all $k$, hence all the $v_{i_{k}}$ are equal. Assume now that there is a $k$ such that $R_{i_{k} i_{k+1}}<0$. Then $v_{i_{k+1}}-v_{i_{k}}<0$, which is a contradiction. Therefore,

$$
\forall k, R_{i_{k} i_{k+1}}=0,
$$

which proves the cyclical consistency of matrix $R$, that is (i).

\subsection{Pareto efficient allocation of indivisible goods}

We now turn to the problem of allocation of indivisible goods, which was initially studied by Shapley and Scarf (1974). Consider $n$ indivisible goods (eg. houses) $j=1, \ldots, n$ to be allocated to $n$ individuals. Cost of allocating (eg. transportation cost) house $j$ to individual $i$ is $c_{i j}$. An allocation is a permutation $\sigma$ of the set $\{1, . ., n\}$ such that individual $i$ gets house $j=\sigma(i)$. Let $S$ be the set of such permutations. We assume for the moment that the initial allocation is given by the identity permutation: good $i$ is allocated to individual $i$. The problem here is to decide whether this allocation is efficient in a Pareto sense.

If there are two individuals, say $i_{1}$ and $i_{2}$ that would both benefit from swapping houses (strictly for at least one), then this allocation is not efficient, as the swap would improve on the welfare of both individuals. Thus if allocation is efficient, then inequalities $c_{i_{1} i_{2}} \leq c_{i_{1} i_{1}}$ and 
$c_{i_{2} i_{1}} \leq c_{i_{2} i_{2}}$ cannot hold simultaneously unless they are both equalities. More generally, Pareto efficiency rules out the existence of exchange rings whose members would benefit (strictly for at least one) from a circular trade. In fact, we shall argue that this problem is dual to the problem of Generalized Revealed Preferences.

\subsection{A dual interpretation of revealed preference}

Let us now formalize the notion of efficient allocation in the previous discussion. Allocation $\sigma_{0}(i)=i$ is Pareto efficient if and only if for any $\sigma \in S$, inequalities

$$
c_{i \sigma(i)} \leq c_{i i}
$$

cannot hold simultaneously unless these are all equalities. By the decomposition of a permutation into cycles, we see that this definition is equivalent to the fact that for every "trading cycle" $i_{1}, \ldots, i_{p+1}=i_{1}$,

$$
\forall k, c_{i_{k} i_{k+1}} \leq c_{i_{k} i_{k}} \text { implies } \forall k, c_{i_{k} i_{k+1}}=c_{i_{k} i_{k}}
$$

that is, introducing $R_{i j}=c_{i j}-c_{i i}$,

$$
\forall k, R_{i_{k} i_{k+1}} \leq 0 \text { implies } \forall k, R_{i_{k} i_{k+1}}=0 \text {, }
$$

which is to say that allocation is efficient if and only if the matrix $R_{i j}$ is cyclically consistent.

By the equivalence between (i) and (ii) in Theorem 0 above, we have the following statement:

Proposition 1. In the housing problem, allocation $\sigma_{0}(i)=i$ is efficient if and only if

$$
\exists v_{i} \text { and } \lambda_{i}>0, v_{j}-v_{i} \leq \lambda_{i} R_{i j}
$$

Before giving an interpretation of this result, we would like to understand the link between efficiency and equilibrium in the housing problem. Assume we start from allocation $\sigma_{0}(i)=i$, and we let people trade. Given a price system $\pi$ where $\pi_{i}$ is the price of house $i$, we assume that individual $i$ can sell her house for price $\pi_{i}$, and therefore can afford any house $j$ whose price $\pi_{j}$ is less than $\pi_{i}$. Therefore, individual $i$ 's budget set $B_{i}$ is the set of houses that sell to a price lower than his'

$$
B_{i}=\left\{j: \pi_{j} \leq \pi_{i}\right\}
$$

We can now define the notion of a competitive equilibrium in this setting. Allocation $\sigma \in S$ is an equilibrium supported by price system $\pi$ if for each individual $i, 1)$ house $\sigma(i)$ is weakly preferred by individual $i$ among all houses that she can afford, while 2) house $\sigma(i)$ is strictly preferred by $i$ among all houses she can strictly afford (i.e., that trade for prices strictly less 
than her house $i$ ). While condition 1) is a necessary requirement, refinement 2) is natural from a behavioral point of view as it implies that if $i$ is indifferent between two houses, then she is going to choose the cheapest house of the two.

In particular, $\sigma_{0}(i)=i$ is a No-Trade equilibrium if there is a system of prices $\pi$, where $\pi_{j}$ is the price of house $j$, such that: 1) whenever house $j$ is affordable for individual $i$, then it is not strictly preferred by $i$ to $i$ 's house, that is $\pi_{j} \leq \pi_{i}$ implies $c_{i j} \geq c_{i i}$, and 2) for any house $j$ in the strict interior of $i$ 's budget set, i.e. that trades for strictly cheaper than house $i$, then individual $i$ strictly prefers her own house $i$ to house $j$, that is $\pi_{j}<\pi_{i}$ implies $c_{i j}>c_{i i}$.

Hence, $\sigma_{0}(i)=i$ is a No-Trade equilibrium supported by prices $\pi$ when conditions (E1) and (E2) below are met, where:

(E1) if house $j$ can be afforded by $i$, then individual $i$ does not strictly prefer house $j$ to house $i$, that is,

$$
\pi_{j} \leq \pi_{i} \text { implies } c_{i j} \geq c_{i i}
$$

that is

$$
\pi_{j} \leq \pi_{i} \text { implies } R_{i j} \geq 0
$$

that is, yet equivalently:

$$
R_{i j}<0 \text { implies } \pi_{j}>\pi_{i} \text {. }
$$

(E2) if house $j$ is (strictly) cheaper than house $i$, then individual $i$ strictly prefers house $i$ to house $j$, that is

$$
\pi_{j}<\pi_{i} \text { implies } c_{i j}>c_{i i}
$$

that is

$$
\pi_{j}<\pi_{i} \text { implies } R_{i j}>0
$$

that is, yet equivalently:

$$
R_{i j} \leq 0 \text { implies } \pi_{j} \geq \pi_{i}
$$

Proposition 2. In the housing problem, allocation $\sigma_{0}(i)=i$ is a No-Trade equilibrium supported by prices $\pi$ if and only if

$$
\begin{aligned}
& R_{i j}<0 \text { implies } \pi_{j}>\pi_{i}, \text { and } \\
& R_{i j} \leq 0 \text { implies } \pi_{j} \geq \pi_{i} .
\end{aligned}
$$

But (EQUILIBRIUM) is exactly formulation (iii) of Theorem 0 with $\pi_{i}=-v_{i}$. By Theorem 0, we know that this statements is equivalent to statement (PARETO). Hence, we get an interpretation of the Generalized Afriat's Theorem as a second welfare theorem

$$
(\text { EQUILIBRIUM) } \Longleftrightarrow(\text { PARETO), }
$$

which we summarize in the following proposition: 
Proposition 3. In the allocation problem of indivisible goods, Pareto allocations are no-trade equilibria supported by prices, and conversely, no-trade equilibria are Pareto efficient.

This is a "dual" interpretation of revealed preference, where $v_{i}$ (utilities in generalized RP theory) become budgets here, and $c_{i j}$ (budgets in generalized RP theory) become utilities here. To summarize this duality, let us give the following table:

\begin{tabular}{||l||l|l||}
\cline { 2 - 3 } \multicolumn{1}{c||}{} & Revealed prefs. & Pareto indiv. allocs. \\
\hline \hline setting & consumer demand & allocation problem \\
\hline \hline budget sets & $\left\{j: c_{i j} \leq c_{i i}\right\}$ & $\left\{-v:-v \leq-v_{i}\right\}$ \\
\hline \hline cardinal utilities to $j$ & $v_{j}$ & $-c_{i j}$ \\
\hline \hline \# of consumers & one & $n, i \in\{1, \ldots, n\}$ \\
\hline \hline \# of experiments & $n$ & one \\
\hline \hline unit of $c_{i j}$ & dollars & utils \\
\hline \hline unit of $v_{i}$ & utils & dollars \\
\hline \hline interpretation & Afriat's theorem & Welfare theorem \\
\hline \hline
\end{tabular}

The table suggests that there is a single agent facing $n$ choices in revealed preference theory, while there are $n$ agents facing one choice in the housing probem. In revealed preference theory, the cardinal utility of alternative $j$ of the consumer in revealed preference theory is $v_{j}$, while $c_{i j}$ expresses a budget set. Conversely, in the housing problem, $-c_{i j}$ is the cardinal utlity consumer $i$ for alternative $j$, while $-v_{j}$ is interpreted as the price of bundle $j$. Hence, the roles of "prices" and "utilities" are exchanges in the two problems, which motivates our contention that Revealed Preference theory and the Housing problem are "dual".

It is worth remarking that the notion of domination used in our definition of Pareto efficiency is different from the one used by Shapley and Scarf. Following a common use, our notion allows for weak domination for some individual, while Shapley and Scarf require strict domination for everybody. The two notions are not equivalent in a setting with indivisible goods. Indeed, using their notion of domination, Shapley and Scarf provide an example in which a Pareto efficient allocation cannot be sustained as a competitive allocation

\subsection{A characterization of rationalizability}

The connection of both the revealed preference problem and the housing problem with assignment problems is clear; we shall now show that there is a useful connection with the optimal assignment problem (recalled below), where the sum of the individual utilities is maximized.

Recall that the data are called rationalizable there are scalars $v_{i}$ such that

$$
\begin{aligned}
& R_{i j}=g_{i}\left(x_{j}\right)<0 \text { implies } v_{j}<v_{i}, \text { and } \\
& R_{i j}=g_{i}\left(x_{j}\right) \leq 0 \text { implies } v_{j} \leq v_{i} .
\end{aligned}
$$


With this in mind, we have the following novel characterization of rationalizability of the data, which extends Theorem 0 :

Theorem 4. In the revealed preference problem, the data are rationalizable if and only if there exist weights $\lambda_{i}>0$ such that

$$
\min _{\sigma \in S} \sum_{i=1}^{n} \lambda_{i} R_{i \sigma(i)}=0
$$

that is

$$
\min _{\sigma \in S} \sum_{i=1}^{n} \lambda_{i} c_{i \sigma(i)}=\sum_{i=1}^{n} \lambda_{i} c_{i i}
$$

Proof of Theorem 4 Start from (4): $\exists \lambda_{i}>0, \min _{\sigma \in S} \sum_{i=1}^{n} \lambda_{i} R_{i \sigma(i)}=0$

$\Longleftrightarrow \exists \lambda_{i}>0, \min _{\sigma \in S} \sum_{i=1}^{n} \lambda_{i} R_{i \sigma(i)}$ is reached for $\sigma=I d$

This problem is to find the assignment $\sigma \in S$ which minimizes the utilitarian welfare loss computed as the sum of the individual costs $K_{i j}=\lambda_{i} R_{i j}$, setting weight one to each individuals. This problem is therefore

$$
\min _{\sigma \in S} \sum_{i=1}^{n} K_{i \sigma(i)}
$$

This problem was reformulated as a Linear Programming problem by Dantzig in the 1930s; see Shapley and Shubik 1971 for a game-theoretic interpretation, and Gretsky, Ostroy and Zame 1992 for the continuous limit), and by the standard Linear Programming duality of the optimal assignment problem (Dantzig 1939; Shapley-Shubik 1971; see Ekeland 2010 for a recent review using the theory of Optimal Transportation)

$$
\min _{\sigma \in S} \sum_{i=1}^{n} K_{i \sigma(i)}=\max _{u_{i}+v_{j} \leq K_{i j}} \sum_{i=1}^{n} u_{i}+\sum_{j=1}^{n} v_{j}
$$

where $S$ is the set of permutations of $\{1, \ldots, n\}$. It is well-known that for a $\sigma_{0} \in S$ solution to the optimal assignment problem, there is a pair $(u, v)$ solution to the dual problem such that

$$
\begin{aligned}
& u_{i}+v_{j} \leq K_{i j} \\
\text { if } j=\sigma_{0}(i), & \text { then } u_{i}+v_{j}=K_{i j} .
\end{aligned}
$$

Hence, (4)

$$
\begin{aligned}
& \Longleftrightarrow \exists \lambda_{i}>0, u, v \in \mathbb{R}^{n} \\
& \begin{array}{l}
u_{i}+v_{j} \leq \lambda_{i} R_{i j} \\
u_{i}+v_{i}=0
\end{array}
\end{aligned}
$$


$\Longleftrightarrow \exists \lambda_{i}>0, v \in \mathbb{R}^{n}$

$$
v_{j}-v_{i} \leq \lambda_{i} R_{i j},
$$

which is (ii) in Theorem 0.

The previous result leads to the following two remarks.

First, recall that matrix $\left(M_{i j}\right)$ is called cyclically monotone if for any cycle $\left(i_{1}, \ldots, i_{p}\right)$, one has

$$
\sum_{k=1}^{p} M_{i_{k} i_{k+1}}-M_{i_{k} i_{k}} \geq 0 .
$$

It is well known (see e.g. Villani, 2003) that cyclical monotonicity is equivalent to (3). Therefore, Theorem (4) states that $\left(R_{i j}\right)$ is cyclically consistent if and only if there are weights $\lambda_{i}>0$ such that $\lambda_{i} R_{i j}$ is cyclically monotone.

As a second remark, introduce

$$
\mathcal{F}=\operatorname{conv}\left(\left(c_{i \sigma(i)}\right)_{i=1, \ldots, n}: \sigma \in S\right) \subset \mathbb{R}^{n}
$$

which is a convex polytope. Note that

$$
\min _{\sigma \in S} \sum_{i=1}^{n} \lambda_{i} c_{i \sigma(i)}=\min _{C \in \mathcal{F}} \sum_{i=1}^{n} \lambda_{i} C_{i} .
$$

We have the following geometric characterization of the fact that the data are rationalizable:

Proposition 5. The data are rationalizable if and only if 0 is an extreme point of $\mathcal{F}$ with a componentwise positive vector in the normal cone.

Proof. Introduce $\mathcal{W}(\lambda)=\min _{C \in \mathcal{F}} \sum_{i=1}^{n} \lambda_{i} C_{i}$. This concave function is the support function of $C . C_{i}^{0}=c_{i \sigma_{0}(i)}$ is an extreme point of $\mathcal{F}$ with a componentwise positive vector in the normal cone if and only 0 is in the superdifferential of $\mathcal{W}$ at such a vector $\lambda$. This holds if and only if $\mathcal{W}(\lambda)=\sum_{i=1}^{n} \lambda_{i} C_{i}^{0}$. 


\section{Strong and weak rationalizability}

\subsection{Indices of rationalizability}

Rationalizability of the data by a single consumer, as tested by Afriat's inequalities, is an important empirical question. Hence it is of interest to introduce measures of how close the data is from being rationalizable. Since Afriat's original work on the topic, many authors have set out proposals to achieve this. Afriat's original "efficiency index" is the largest $e \leq 1$ such that $R_{i j}^{e}=R_{i j}+(1-e) b_{i}$ is cyclically consistent, where $b_{i}>0$ is a fixed vector with positive components. In Afriat's setting, $R_{i j}=x_{j} \cdot p_{i}-x_{i} \cdot p_{i}$, and $R_{i j}^{e}=x_{j} \cdot p_{i}-e x_{i} \cdot p_{i}$, so $b_{i}=x_{i} \cdot p_{i}$. Many other tests and empirical approaches have followed and are discussed in Varian's (2006) review paper. A recent proposal is given in Echenique, Lee and Shum (2011), who introduce the "money pump index" as the amount of money that could be extracted from a consumer with non-rationalizable preferences.

In the same spirit, we shall introduce indices that will measure how far the dataset is from being rationalizable. The indices we shall build are connected to the dual interpretation of revealed preferences we have outlined above. Our measures of departure from rationalizability will be connected to measures of departure from Pareto efficiency in that problem. One measure of departure from efficiency is the welfare gains that one would gain from moving to the efficient frontier; this is interpretable as Debreu's (1951) coefficient of resource utilization!, in the case of a convex economy. This is only an analogy, as we are here in an indivisible setting, but this exactly the idea we shall base the construction of our indices on.

It seems natural to introduce index $A$ as

$$
A=\max _{\lambda \in \Delta} \min _{\sigma \in S} \sum_{i=1}^{n} \lambda_{i} R_{i \sigma(i)}
$$

where $\Delta=\left\{\lambda \geq 0, \sum_{i=1}^{n} \lambda_{i}=1\right\}$ is the simplex of $\mathbb{R}^{n}$. Indeed, we have

$$
A \leq 0,
$$

and by compacity of $\Delta$, equality holds if and only if there exist $\lambda \in \Delta$ such that

$$
\min _{\sigma \in S} \sum_{i=1}^{n} \lambda_{i} R_{i \sigma(i)}=0 .
$$

Of course, this differs from our characterization of rationalizability in Theorem 4 as there the weights $\lambda_{i}$ 's need to be all positive, not simply nonnegative. It is easy to construct examples where (4) holds with some zero $\lambda_{i}$ 's and $\sigma_{0}(i)=i$ is not efficient. For example, in the housing

\footnotetext{
${ }^{1}$ We thank Don Brown for suggesting this interpretation to us.
} 
problem, if individual $i=1$ has his most preferred option, then $\lambda_{1} \neq 0$ and all the other $\lambda_{i}$ 's are zero, and $A=0$, thus (4) holds. However, allocation may not be Pareto because there may be inefficiencies among the rest of the individuals.

Hence imposing $\lambda>0$ is crucial. Fortunately, it turns out that one can restrict the simplex $\Delta$ to a subset which is convex, compact and away from zero, as shown in the next lemma.

Lemma 6. There is $\varepsilon>0$ (dependent only on the entries of matrix $c$, but independent on the matching considered) such that the following disjuction holds

- either there exist no scalars $\lambda_{i}>0$ satisfying the condtions in Theorem 4

- or there exist scalars $\lambda_{i}>0$ satisfying the condtions in Theorem 4 and such that

$$
\left\{\begin{array}{c}
\lambda_{i} \geq \varepsilon \text { for all } i \\
\sum_{i=1}^{n} \lambda_{i}=1 .
\end{array}\right.
$$

Proof. Standard construction (see (Fostel et al. (2004) $)$ ) of the $\lambda_{i}$ 's and the $v_{i}$ 's provides a deterministic procedure that returns strictly positive $\lambda_{i} \geq 1$ within a finite and bounded number of steps, with only the entries of $R_{i j}$ as input; hence $\lambda$, if it exists, is bounded, so there exists $M$ depending only on $R$ such that $\sum_{i=1}^{n} \lambda_{i} \leq M$. Normalizing $\lambda$ so that $\sum_{i=1}^{n} \lambda_{i}=1$, one sees that one can choose $\varepsilon=1 / M$.

We denote $\Delta_{\varepsilon}$ the set of such vectors $\lambda$, and we recall that $\Delta$ is the set of $\lambda$ such that $\lambda_{i} \geq 0$ for all $i$ and $\sum_{i=1}^{n} \lambda_{i}=1$. Recall $R_{i j}=c_{i j}-c_{i i}$, and introduce

$$
A^{*}=\max _{\lambda \in \Delta_{\varepsilon}} \min _{\sigma \in S} \sum_{i=1}^{n} \lambda_{i} R_{i \sigma(i)},
$$

so that we have

$$
A^{*} \leq 0
$$

and equality if and only if the data are rationalizable (as in this case there exist $\lambda_{i}>0$ such that the characterization of rationalizability in 4 is met). Further, as $\Delta_{\varepsilon} \subset \Delta$, one gets

$$
\underbrace{\max _{\lambda \in \Delta_{\varepsilon}} \min _{\sigma \in S} \sum_{i=1}^{n} \lambda_{i} R_{i \sigma(i)}}_{A^{*}} \leq \underbrace{\max _{\lambda \in \Delta} \min _{\sigma \in S} \sum_{i=1}^{n} \lambda_{i} R_{i \sigma(i)}}_{A} \leq 0 .
$$


The max-min formulation for index $A$ leads naturally to introduce a new index which comes from the dual min-max program

$$
\begin{aligned}
B & =\min _{\sigma \in S} \max _{\lambda \in \Delta} \sum_{i=1}^{n} \lambda_{i} R_{i \sigma(i)} \\
& =\min _{\sigma \in S} \max _{i \in\{1, \ldots, n\}} R_{i \sigma(i)}
\end{aligned}
$$

Note that the inequality max min $\leq \min \max$ always hold, so $A \leq B$, and further, we have

$$
B \leq \max _{i \in\{1, \ldots, n\}} R_{i i}=0 .
$$

Therefore we have

$$
\underbrace{\max _{\lambda \in \Delta_{\varepsilon}} \min _{\sigma \in S} \sum_{i=1}^{n} \lambda_{i} R_{i \sigma(i)}}_{A^{*}} \leq \underbrace{\max _{\lambda \in \Delta} \min _{\sigma \in S} \sum_{i=1}^{n} \lambda_{i} R_{i \sigma(i)}}_{A} \leq \underbrace{\min _{\sigma \in S} \max _{\lambda \in \Delta} \sum_{i=1}^{n} \lambda_{i} R_{i \sigma(i)}}_{B} \leq 0 .
$$

We shall come back to the interpretation of $A^{*}=0, A=0$ and $B=0$ as stronger or weaker forms of rationalizability of the data. Before we do that, we summarize the above results.

Proposition 7. We have:

(i) $A^{*}=0$ if and only if there exist scalars $v_{i}$ and weights $\lambda_{i}>0$ such that

$$
v_{j}-v_{i} \leq \lambda_{i} R_{i j}
$$

(ii) $A=0$ if and only if there exist scalars $v_{i}$ and weights $\lambda_{i} \geq 0$, not all zero, such that

$$
v_{j}-v_{i} \leq \lambda_{i} R_{i j}
$$

(iii) $B=0$ if and only if

$$
\forall \sigma \in S, \exists i \in\{1, \ldots, n\}: R_{i \sigma(i)} \geq 0 .
$$

(iv) One has

$$
A^{*} \leq A \leq B \leq 0
$$

Note that it is easy to find examples where these inequalities hold strict for $n \geq 3$.

Proof. (i) follows from Lemma 6, To see (ii), note that $A=0$ is equivalent to the existence of $\lambda \in \Delta$ such that $\min _{\sigma \in S} \sum_{i=1}^{n} \lambda_{i} R_{i \sigma(i)}=0$, and the rest follows from Theorem 4 . The condition in (iii) is equivalent to the fact that for all $\sigma \in S, \max _{i \in\{1, \ldots, n\}} R_{i \sigma(i)} \geq 0$, that is for all $\sigma \in S$, there exists $i \in\{1, \ldots, n\}$ such that $R_{i \sigma(i)} \geq 0$. The inequalities in (iv) were explained above. 
In an unpublished manuscript ( (Geanakoplos (2006) $)$ ) that he kindly communicated to us on our request, John Geanakoplos introduces the following minmax problem, which in our notations can be defined as

$$
G=\max _{\lambda \in \Delta} \min _{\sigma \in C} \sum_{i=1}^{n} \lambda_{i} R_{i \sigma(i)}
$$

where $C \subset S$ is the set of permutations that have only one cycle, ie. such that there exist a cycle $i_{1}, \ldots, i_{p+1}=i_{1}$ such that $\sigma\left(i_{k}\right)=i_{k+1}$ and $\sigma(i)=i$ if $i \notin\left\{i_{1}, \ldots, i_{p}\right\}$. As $C \subset S$ one has

$$
A \leq G \leq 0
$$

Geanakoplos uses index $G$ and von Neuman's minmax theorem in order to provide an interesting alternative proof of Afriat's theorem. However, it seems that index $G$ is not directly connected with the assignment problem.

\subsection{Interpretation of indexes $A^{*}, A, B$}

As seen above, the index $A^{*}$ was constructed so that $A^{*}=0$ if and only if the data are rationalizable. The indexes $A$ and $B$ will both be equal to 0 if the data are rationalizable; hence $A<0$ or $B<0$ imply that the data is not rationalizable; however the converse is not true, so these indexes can be interpreted as measures of weaker form of rationalizability. Hence we would like to give a meaningful interpretation of the situations where $A=0$ and $B=0$. It turns out that $A=0$ is equivalent to the fact that a subset of the observations can be rationalized, the subset having to have a property of coherence that we now define. $B=0$ is equivalent to the fact that one cannot partition the set of observations into increasing cycles, a notion we will now define. In other words, the condition $B<0$ means that any observation is part of a preference cycles-indeed a very strong departure from rationalizability. These indexes may be used to compute identified regions in models with partial identification; see Ekeland, Galichon and Henry (2010) and references therein.

Throughout this subsection it will be assumed that no individual is indifferent between two distinct consumptions for the direct revealed preference relation, that is:

Assumption A. In this subsection, $R$ is assumed to verify $R_{i j} \neq 0$ for $i \neq j$.

We first define the notion of coherent subset of observations.

Definition 8 (Coherent subset). In the revealed preference problem, a subset of observations included in $\{1, \ldots, n\}$ is said to be coherent when $i \in I$ and $i$ directly revealed preferred to $j$ implies $j \in I$. Namely, I is coherent when

$$
i \in I \text { and } R_{i j}<0 \text { implies } j \in I \text {. }
$$


In particular, $\{1, \ldots, n\}$ is coherent; any subset of observations where each observation is directly revealed preferred to no other one is also coherent. Next, we define the notion of increasing cycles.

Definition 9 (Increasing cycles). A cycle $i_{1}, \ldots, i_{p+1}=i_{1}$ is called increasing when each observation is strictly directly revealed preferred to its predecessor. Namely, cycle $i_{1}, \ldots, i_{p+1}=i_{1}$ is increasing when

$$
R_{i_{k} i_{k+1}}<0 \text { for all } k \in\{1, \ldots, p\}
$$

Of course, the existence of an increasing cycle implies that the matrix $R$ is not cyclically consistent, hence it implies in the revealed preference problem that the data are not rationalizable. It results from the definition that an increasing cycle has length greater than one.

We now state the main result of this section, which provides an economic interpretation for the indexes $A^{*}, A$ and $B$.

\section{Theorem 10. We have:}

(i) $A^{*}=0$ iff the data are rationalizable,

(ii) $A=0$ iff a coherent subset of the data is rationalizable,

(iii) $B=0$ iff there is no partition of $\{1, \ldots, n\}$ in increasing cycles.

and (i) implies (ii), which implies (iii).

Proof. (i) was proved in Theorem 4 above.

Let us show the equivalence in $(i i)$. From Proposition $7 A=0$ is equivalent to the existence of $\exists \lambda_{i} \geq 0, \sum_{i=1}^{n} \lambda_{i}=1$ and $v \in \mathbb{R}^{n}$ such that

$$
v_{j}-v_{i} \leq \lambda_{i} R_{i j}
$$

so defining $I$ as the set of $i \in\{1, \ldots, n\}$ such that $\lambda_{i}>0$, this implies a subset $I$ of the data is rationalizable. We now show that $I$ is coherent. Indeed, for any two $k$ and $l$ not in $I$ and $i$ in $I$, one has $v_{k}=v_{l^{\prime}} \geq v_{i}$; thus if $i \in I$ and $R_{i j}<0$, then $v_{j}<v_{i}$, hence $j \in I$, which show that $I$ is coherent.

Conversely, assume a coherent subset of the data $I$ is rationalizable. Then there exist $\left(u_{i}\right)_{i \in I}$ and $\left(\mu_{i}\right)_{i \in I}$ such that $\mu_{i}>0$ and

$$
u_{j}-u_{i} \leq \mu_{i} R_{i j}
$$

for $i, j \in I$. Complete by $u_{i}=\max _{k \in I} u_{k}$ for $i \notin I$, and introduce $\tilde{R}_{i j}=1_{\{i \in I\}} R_{i j}$. One has $\tilde{R}_{i j}<0$ implies $i \in I$ and $R_{i j}<0$ hence $j \in I$ by the coherence property of $I$, thus $u_{j}-u_{i}<0$. 
Now $\tilde{R}_{i j}=0$ implies either $i \notin I$ or $R_{i j}=0$ thus $i=j$; in both cases, $u_{j} \leq u_{i}$. Therefore, one has

$$
\begin{aligned}
& \tilde{R}_{i j}<0 \text { implies } u_{j}-u_{i}<0, \text { and } \\
& \tilde{R}_{i j} \leq 0 \text { implies } u_{j}-u_{i} \leq 0 .
\end{aligned}
$$

Hence by Theorem 0 , there exist $v_{i}$ and $\bar{\lambda}_{i}>0$ such that

$$
v_{j}-v_{i} \leq \bar{\lambda}_{i} \tilde{R}_{i j}
$$

and defining $\lambda_{i}=\bar{\lambda}_{i} 1_{\{i \in I\}}$, one has

$$
v_{j}-v_{i} \leq \lambda_{i} R_{i j}
$$

which is equivalent to $A=0$.

(iii) Now Proposition 7 implies that $B<0$ implies that there is $\sigma \in S$ such that $\forall i \in$ $\{1, \ldots, n\}, R_{i \sigma(i)}<0$. The decomposition of $\sigma$ in cycles gives a partition of $\{1, \ldots, n\}$ in increasing cycles.

(iv) The implication $(i) \Rightarrow(i i) \Rightarrow($ iii $)$ results from inequality $A^{*} \leq A \leq B \leq 0$.

\section{Concluding remarks}

To conclude, we make a series or remarks.

First, we have shown how our dual interpretation of Afriat's theorem in its original Revealed Preference context as well as in a less traditional interpretation of efficiency in the housing problem could shed new light and give new tools for both problems. It would be interesting to undestand if there is a similar duality for problems of revealed preferences in matching markets, recently investigated by Galichon and Salanié (2010) and Echenique et al. (2011).

Also, we argue that it makes sense to investigate "weak rationalizability" (ie. $A=0$ or $B=0$ ) instead of "strong rationalizability" (ie. $A^{*}=0$ ), or equivalently, it may make sense to allow some $\lambda_{i}$ 's to be zero. In the case of $A=0$, recall the $\lambda_{i}$ 's are interpreted in Afriat's theory as the Lagrange multiplier of the budget constraints. Allowing for $\lambda=0$ corresponds to excluding wealthiest individuals as outliers. It is well-known that when taken to the data, strong rationalizability is most often rejected. It would be interesting to test econometrically for weak rationalizability, namely whether $A=0$.

$B<0$ is a very strong measure of nonrationalizability, as $B<0$ means that one can find a partition of the observation set into increasing cycles, which seems a very strong violations 
of the Generalized Axiom of Revealed Preference. But this may arise in some cases, especially with a limited number of observations.

We should emphasize on the fact that indexes $A^{*}, A$ and $B$ provide a measure of how close the data is from being rationalizable, in the spirit of Afriat's efficiency index. It is clear for every empirical researcher that the relevant question about revealed preference in consumer demand is not whether the data satisfy GARP, it is how much they violate it. These indexes are an answer to that question. Also the geometric interpretation of rationalizable is likely to provide useful insights for handling unobserved heterogeneity. We plan to investigate this question in further research.

Last, it seems that an interesting research avenue (not pursued in the present paper) deals with exploring similar connections as in this paper, and understand what would be the analogue of variants of the theories discussed here. For instance, in the light of the duality exposed in the present paper, it would be interesting to characterize the dual theory to the Revealed Preference theory for collective models of household consumption as in Cherchye, De Rock and Vermeulen (2007, 2010). Similarly, it would also be of interest to understand the Revealed Preference dual of the two-sided version of the housing problem, namely the marriage problem, where both sides of the market have preferences over the other side. In these two cases as in others, what becomes of the connection stressed in the current paper? this question, too, is left for future research.

\section{References and Notes}

Afriat (1967).

Afriat, S. (1967). "The construction of a Utility Function from Demand Data", International Economic Review 8, 67-77.

Chercheye, De Rock, and Vermeulen (2007). Cherchye, L., De Rock, B., and Vermeulen, F. (2007). "The collective model of household consumption: A nonparametric characterization", Econometrica 75, pp. 553-574.

Chercheye, De Rock, and Vermeulen (2010). Cherchye, L., De Rock, B., and Vermeulen, F. (2010). "An Afriat theorem for the collective model of household consumption," Journal of Economic Theory 145, pp.1142-1163.

Diewert (1973).

Diewert, W. (1973). "Afriat and Revealed Preference Theory”, Review of Economic Studies 40, 419-425. 
Debreu (1951).

Echenique et al. (2011).

Echenique, Lee and Shum (2011).

Ekeland (2010).

Ekeland, Galichon and Henry (2010).

Forges and Minelli (2009).

Fostel et al. (2004).

Galichon and Salanié (2010).

Geanakoplos (2006).

Gretsky et al. (1992).

Houthakker (1950).
Debreu, G. (1951). "The Coefficient of Resource Utilization," Econometrica, 19, 273-292.

Echenique, F. Lee, S.M., Shum, M., and Yenmez, B. (2011). "The Revealed Preference Theory of Stable and Extremal Stable Matchings". Forthcoming, Econometrica.

Echenique, F., Lee, S.M., and Shum, M. (2011). "The Money Pump As a Measure of Revealed Preference Violations," Journal of Political Economy 119, pp. 1201-1223.

Ekeland, I. (2010). "Notes on Optimal Transportation," Economic Theory 42 (2), pp. 437459.

Ekeland, I., Galichon, A. and Henry, M. (2010). "Optimal Transportation and the falsifiability of incompletely specified models", Economic Theory 42 (2), pp. 355-374.

Forges, F. and Minelli, E. (2009). "Afriat's Theorem for General Budget Sets," Journal of Economic Theory 144, 135-145.

Fostel, A., H. Scarf and M. Todd (2004). "Two New Proofs of Afriat's Theorem", Economic Theory 24, 211-219.

Galichon, A., and Salanié, B. (2010). "Cupid's Invisible Hands," working paper.

Geanakoplos, J. (2006). "Afriat from MaxMin," unpublished manuscript, private communication.

Gretsky, N., Ostroy, J. and Zame, W. (1992). "The Nonatomic Assignment Model," Economic Theory 2 (1), pp. 103-127.

Houthakker, H., (1950). "Revealed preferences and the utility function". Economica 17, 159174. 
Matzkin (1991).

Roth et al. (2004).

Samuelson (1938).

Shapley and Scarf (1974).

Shapley and Shubik (1972).

Teo and Vohra (2003).

Varian (1982).

Varian (2006).

Villani (2003).
1. Quah, J. (2012). “A Revealed Preference Test for Weakly Separable Preferences". Oxford University discussion paper 601 .

Matzkin, R. (1991). "Axioms of Revealed Preferences for Nonlinear Choice Sets", Econometrica 59, 1779-1786.

Roth, A.E., T. Sönmez, and U. Ünver (2004). "Kidney exchange," Quarterly Journal of Economics, 119, 457-488.

Samuelson, P. (1938). "A Note on the Pure Theory of Consumer's Behavior", Economica NS 5, 61-71.

Shapley, L. and H. Scarf (1974). "On cores and indivisibility," Journal of Mathematical Economics, 1, 23-37.

Shapley, L. and Shubik, M. (1972). "The assignment game I: the core," International Journal of Game Theory 1, pp. 111-130.

Chung-Piaw, T. and Vohra, R. V. (2003). "Afriat's Theorem and Negative Cycles" technical report.

Varian, H. (1982). "The Nonparametric Approach to Demand Analysis". Econometrica 50, 945-973.

Varian, H. (2006). "Revealed Preference". In Samuelsonian Economics and the Twenty-First Century, Michael Szenberg, Lall Ramrattan and Aron A. Gottesman, eds. Oxford University Press, 99-115.

Villani, C. (2003). Topics in Optimal Transportation. AMS. 